\title{
Effect of Rice Fungal Endophytes on Seed Germination and Seedling Growth of Rice
}

\author{
Lalngaihawmi $^{1 *}$, S. Banik ${ }^{2}$, P. Chakruno ${ }^{2}$ and Khatemenla ${ }^{3}$ \\ ${ }^{1}$ Department of Plant Pathology, Assam Agricultural University, Jorhat (785013), \\ Assam, India \\ ${ }^{2}$ Department of Plant Pathology, School of Agricultural Sciences and Rural Development \\ (SASRD), Nagaland University, Medziphema Campus (797106), Nagaland, India \\ ${ }^{3}$ Department of Horticulture, Assam Agricultural University, Jorhat (785013), Assam, India \\ *Corresponding author
}

\begin{abstract}
A B S T R A C T
An investigation was carried out to study the effect of rice fungal endophytes on the seed germination and seedling growth of rice at the department of Plant Pathology, SASRD,

Keywords

Endophytes, Potato

dextrose broth,

Culture filtrate,

Blotter method

Article Info

Accepted:

30 March 2018

Available Online:

10 April 2018

Medziphema campus, Nagaland. Culture filtrate of fourteen fungal endophytes viz. Cladosporium cladosporioides, Penicillium citrinum, Fusarium moniliforme, Trichoderma asperellum, Penicillium pinophilum, Aspergillus niger, Aspergillus flavus, Drechslera specifera, Penicillium oxalicum, Geotrichum candidum, Curvularia lunata, Aspergillus amstelodami, Talaromyces sp., and Chaetomium ochraceum were tested at different concentration $(25,50$ and $100 \%)$ at different dipping periods (15, 30 and 60 minutes). The culture filtrates of the endophytes were prepared by growing the endophytes in Potato Dextrose Broth. Blotter method was used for testing the germination of seeds. The maximum per cent germination of rice seeds was recorded with the endophyte $P$. citrinum (96.65) when dipped for 15 and 60 minutes at 50 and $25 \%$ concentration respectively. The maximum shoot length was recorded with seeds treated with $C$. cladosporioides (13.74 $\mathrm{mm}$ ) when dipped for 30 minutes at $100 \%$ concentration as compared to $6.74 \mathrm{~mm}$ shoot length recorded in control experiments. The maximum root length was recorded with seeds treated with A. amstelodami $(42.59 \mathrm{~mm})$ when dipped for 60 minutes at $100 \%$ concentration of culture filtrate as compared to control (41.19 $\mathrm{mm})$.
\end{abstract}

\section{Introduction}

Endophytes are microorganisms living within plant tissues for all or part of their life cycle without causing any visible symptoms of their presence are defined as endophytes (Wilson, 1993; Saikkonen et al., 2004a). Endophytes inhabit majority of healthy and symptomless plants, in various tissues, seeds, roots, stems and leaves (Johri, 2006). Plants benefit extensively by harbouring these endophytic microbes; they promote plant growth (Compant et al., 2005) and confer enhanced resistance to various pathogens (Clay and Schardl, 2002; Höflich, 2000; Arnold et al., 2003) by producing antibiotics (Ezra et al., 2004). Endophytes also produce unusual secondary metabolites of plant importance 
(Taechowisan et al., 2005). The presence of fungal endophytes can cause higher rates of water loss in leaves (Anonymous, 2009). However, certain fungal endophytes help plant to survive drought and heat. Fungal endophyte-related host benefits are common phenomena, and have been focus of much research, particularly among the grass endophytes (Don, 2011). Endophytic fungi are very common and are well diversified. Every plant species is found to have at least host one fungal endophytes, but usually asymptomatic and sometimes systemically (Faeth and Fagan, 2002).

The possibility that plant associated microbial diversity is influenced by the diversity of plant species and environmental factors suggest a greater potential for harvesting unique potential secondary metabolites from endophytic microorganisms found in association with hitherto unexploited floristically diverse plant communities (Gunatilaka, 2006). Faeth and co-workers have recently isolated and partially identified over 400 endophytic fungal taxa from Arizona fescue (Schulthess and Faeth, 1998), more than 40 species of endophytic fungi from Emory oak (Faeth and Hammon, 1997) and 22 endophyte species from cacti (Suryanarayanan et al.,2005) growing in very dry regions of Arizona. It is noteworthy that endophytically established fungi, such as Hypoxylon spp. undergo active mycelial development in response to water stress in host organs (Chapela, 1989). Even endophytes in agronomic grasses grown under mesic conditions produce metabolites that increase resistance to drought stress (Clay and Holah, 1999; Bush et al., 1997). Endophyti fungi Phomopsis liquidambarican establish a mutualistic symbiotic relationship with rice which in turn promotes the growth and yield of the host plant and reduces the amount of nitrogen $(\mathrm{N})$ fertilizer required for plant growth (Bo et al., 2014). Extracts of plant endophytes have also been found to have significant effect on the germination potential, vigour index of rice seeds seedling dry weight, fresh weight, root length, root dry weight, and the number of tillering ( $\mathrm{Na}$ et al., 2014). Understanding the need to exploit the potential and identification of endophytic fungi on the growth and development of rice this investigation was carried out.

\section{Materials and Methods}

The investigation was carried out in the Department of Plant Pathology, SASRD, Nagaland University, Medziphema Campus, Nagaland. Local cultivar of rice, Kemenya was used for this investigation.

\section{Isolation and identification of fungal endophyte}

The rice plant was surfaced sterilized by sequential emersion in $70 \%$ ethanol for 5 minutes and Sodium hypochloride $(0.9 \%$ available chlorine) for 20 minutes (Tian et al., 2004). Different plant parts viz. leaves, shoots and roots were cut into fragment of $1 \mathrm{~cm}$ size. After which they were placed on PDA (Potato Dextrose Agar- distilled water $1000 \mathrm{ml}$, potato $200 \mathrm{~g}$, dextrose $20 \mathrm{~g}$, agar agar $20 \mathrm{~g}$ ) plates, each plates containing 10 numbers of fragment and were incubated at $25^{\circ} \mathrm{C}$ for $2-3$ days. The fungal colonies thus obtained were further purified and maintained in PDA slants. Identification of all the fungal endophytes isolated in this investigation was made by ITCC (Indian Type Cultural Collection), Division of Plant Pathology IARI (Indian Agricultural Research Institute), New Delhi 110012, basing on their cultural and morphological characteristics.

\section{Preparation of culture filtrate}

The culture filtrates of the endophytes were prepared by growing the endophytes in Potato 
Dextrose Broth (PDB: Peeled Potato 200g, Dextrose $20 \mathrm{~g}$ and Distilled water $1000 \mathrm{ml}$ ) in aseptic condition and were incubated at $25^{\circ} \mathrm{C}$. The mycelium of the fungus was discarded once it covered up the entire broth. The broth was then filtered with the help of sterilized Whatman filter paper no.44 to extrude (100\% concentration) the culture filtrate. The culture filtrates were later used for testing the effectiveness of the fungal endophytes on the rice plant.

\section{Seed germination and seedling growth}

Blotter method was used for testing the germination of seeds, where 30 numbers of seeds were treated with the culture filtrate of each fungal endophyte at different concentrations viz. 25, 50 and $100 \%$ and for different dipping periods i.e. 15, 30 and 60 minutes under each concentration.

Each of the treatment was maintained in three replications of 10 seeds each. Control plates were also maintained by dipping the seeds in sterile distilled water for comparison. The treated seeds along with the seeds dipped in sterile water (control) were then placed separately in moist blotter inside Petri plate and incubated at room temperature $28 \pm 2{ }^{\circ} \mathrm{C}$. The blotters were periodically re-wetted to prevent drying. Seed germination was determined on the fifth day from the treatment of the rice seeds with the endophytes and the number of germinated and un-germinated seeds were recorded and per cent germination of the seeds were calculated using an appropriate formula:

Per cent gemination $=\frac{\text { No, of seeds germinated }}{\text { Total number of seeds }} \times 100$

On the fifth day after the treatment of the seeds with the culture filtrate of the endophytes. The length of the shoots as well as the length of the roots of each germinated seeds were measured separately and were recorded. Measurements of the seedlings from the control plates were also recorded.

\section{Statistical analysis}

Significance of different sources of variance was tested by Error mean square using Snedor's ' $F$ ' test of probability at $5 \%$ level of significance. The percentage values were transformed into corresponding values by Arc sine and square root transformation.

\section{Results and Discussion}

Altogether 14 numbers of fungal endophytes of rice were isolated and identified in this investigation viz. Cladosporium cladosporioides, Penicillium citrinum, Fusarium moniliforme, Trichoderma asperellum, Penicillium pinophilum, Aspergillus niger, Aspergillus flavus, Drechslera specifera, Penicillium oxalicum, Geotrichum candidum, Curvularia lunata, Aspergillus amstelodami, Talaromyces sp., and Chaetomium ochraceum.

Effect of culture filtrate of fungal endophytes of rice on rice seed germination $(\%)$ in vitro

Significant influence on the germination percentage of rice seeds were observed for different concentration of culture filtrate of fungal endophytes viz. 25, 50 and $100 \%$ at three different dipping periods viz. 15, 30 and 60 minutes as depicted in Table 1. The maximum per cent germination of rice seeds was recorded with the endophyte $P$. citrinum (96.65) when dipped for 15 and 60 minutes at 50 and $25 \%$ concentration respectively and $P$. pinophillum when dipped for 30 minutes at $50 \%$ concentration as compared to $86.67 \%$ germination recorded in control experiments. The least percent germination of rice seeds was recorded with the endophyte $P$. oxalicum 
(70) at 50 and $100 \%$ concentration of culture filtrate dipped for 60 minutes each and $T$. asperellum at $100 \%$ concentration dipped for 15 minutes. The highest mean percent germination was recorded in the rice seeds dipped in culture filtrate of A. niger (88.52) and the least mean percent germination was recorded in the rice seeds dipped in that of $P$. oxalicum (75.93) as compared to control that recorded $85.56 \%$ germination. Whereas, with respect to time and concentration, irrespective of the different culture filtrates of endophytes applied, the rice seeds dipped in 100\% concentration of culture filtrate for 30 minutes recorded the highest mean percent germination $(84.64 \%)$ and the least mean percent germination $(82.00 \%)$ was recorded for rice seeds dipped in $100 \%$ concentration culture filtrates dipped for 15 minutes.

However, it was observed that different concentrations of the fungal endophytes had no significant difference of on the per cent germination of rice at different time intervals. But treatments with fungal endophyte species had significant influence on per cent germination while interactions between treatments applied and concentrations of the culture filtrate does not have significant effect on the per cent germination except for those rice seeds which were dipped in culture filtrates for 30 minutes.

The findings that describe the effect of fungal endophytes especially the endophytic species tested in the present investigation on germination of rice seeds has not been reported earlier. However, Lin et al., (2007) reported that germination of rice seeds infected with endophytic Phomopsis sp. isolated from the inner bark of Bischofia polycarpa was significantly greater than that of endophyte free plants. Cheplick et al., (1989) and Clay (1987) reported that seeds of tall fescue and perennial ryegrass germinate more rapidly and to higher levels when infected with endophytes. Though in the present investigation, the rapidity of germination was not recorded, the percent germination at higher levels has been achieved by some endophytes like $P$. citrinum and $P$. pinophilum both recording $96.65 \%$ germination of rice seeds when dipped in their culture filtrates compared to $86.67 \%$ germination in control. In the present experiment, rice seeds were dipped in culture filtrates of fungal endophytes that are contrary to the works of Cheplick et al., (1989) and Clay (1987) who infected their planting materials with the endophytes directly. $\mathrm{Na}$ et $a l$. , (2014) reported that germination potential and vigor index of rice seeds treated by mixture of $5 \mathrm{ng} / \mathrm{ml}$ of extracts of Alternaria (R) and $5 \mathrm{ng} / \mathrm{ml}$ of extracts of Coprinus micaceus (D) at germination stage were higher than those of control by $9.52 \%$ and $23.94 \%$. In the present study, percent germination of rice seeds was higher by $10 \%$ (P. citrinum and $P$. pinophilum both recording $96.65 \%$ germination compared to control that is $86.67 \%$ ) due to the influence of fungal endophytes.

Higher per cent germination of rice seeds in presence of culture filtrates of $P$. citrinum and $P$. pinophilum indicates the positive impact of these endophytes on seed health of rice. Endophytes are intricately associated with their host plants in tissue specific manner (Fisher and Petrini, 1992). Endophytes population of rice plant also differ in terms of diversity and quantity at different growth stages of rice plant (Imchen, 2015). It was observed earlier that $P$. pinophilum is specific to seedling stage of rice and not found in vegetative and maturity stage, whereas, $P$. citrinum is more abundant in seedling and vegetative stage of rice plant than maturity stage (Imchen, 2015). Thus, association of this Penicillium spp. with the seedling stage of rice plant perhaps explains their promotion effect on rice seed germination. 
Table.1 Effect of culture filtrate of fungal endophytes of rice on rice seed germination (\%) in vitro

\begin{tabular}{|c|c|c|c|c|c|c|c|c|c|c|c|}
\hline \multirow{3}{*}{\multicolumn{2}{|c|}{ Endophyte species }} & \multicolumn{10}{|c|}{ Per cent germination of rice seeds treated with culture filtrate of fungal endophytes } \\
\hline & & \multicolumn{3}{|c|}{15 mins } & \multicolumn{3}{|c|}{$\mathbf{3 0}$ mins } & \multicolumn{3}{|c|}{ 60mins } & \multirow[t]{2}{*}{ Mean } \\
\hline & & \multicolumn{2}{|r|}{$50 \%$} & \multirow{2}{*}{$\begin{array}{l}\mathbf{1 0 0 \%} \\
83.33 \\
(9.13)\end{array}$} & $25 \%$ & $50 \%$ & \multirow{2}{*}{$\begin{array}{l}\mathbf{1 0 0 \%} \\
86.67 \\
(9.31)\end{array}$} & \multirow{2}{*}{$\begin{array}{c}\mathbf{2 5 \%} \\
90.00 \\
(9.49)\end{array}$} & $\mathbf{5 0 \%}$ & $100 \%$ & \\
\hline 1. & Cladosporium cladosporioides & $\begin{array}{l}89.99 \\
(9.48)^{*}\end{array}$ & $\begin{array}{l}80.00 \\
(8.93)\end{array}$ & & $\begin{array}{l}86.67 \\
(9.31)\end{array}$ & $\begin{array}{l}86.67 \\
(9.31)\end{array}$ & & & $\begin{array}{l}83.33 \\
(9.13)\end{array}$ & $\begin{array}{l}83.33 \\
(9.13)\end{array}$ & 85.55 \\
\hline 2. & Penicillium citrinum & $\begin{array}{l}83.33 \\
(9.13)\end{array}$ & $\begin{array}{l}96.65 \\
(9.83)\end{array}$ & $\begin{array}{l}76.67 \\
(8.75)\end{array}$ & $\begin{array}{l}73.33 \\
(8.56)\end{array}$ & $\begin{array}{l}90.00 \\
(9.49)\end{array}$ & $\begin{array}{l}73.33 \\
(8.56)\end{array}$ & $\begin{array}{l}96.65 \\
(9.83)\end{array}$ & $\begin{array}{l}80.00 \\
(8.94)\end{array}$ & $\begin{array}{l}80.00 \\
(8.93)\end{array}$ & 83.33 \\
\hline 3. & Fusarium moniliforme & $\begin{array}{l}80.00 \\
(8.94)\end{array}$ & $\begin{array}{l}83.33 \\
(9.13)\end{array}$ & $\begin{array}{l}83.33 \\
(9.13)\end{array}$ & $\begin{array}{l}89.99 \\
(9.48)\end{array}$ & $\begin{array}{l}86.67 \\
(9.31)\end{array}$ & $\begin{array}{l}86.67 \\
(9.31)\end{array}$ & $\begin{array}{l}83.33 \\
(9.13)\end{array}$ & $\begin{array}{l}86.67 \\
(9.31)\end{array}$ & $\begin{array}{l}83.33 \\
(9.13)\end{array}$ & 84.81 \\
\hline 4. & Trichoderma asperellum & $\begin{array}{l}76.67 \\
(8.74)\end{array}$ & $\begin{array}{l}76.67 \\
(8.75)\end{array}$ & $\begin{array}{l}70.00 \\
(8.37)\end{array}$ & $\begin{array}{l}83.33 \\
(9.13)\end{array}$ & $\begin{array}{l}73.33 \\
(8.56)\end{array}$ & $\begin{array}{l}86.67 \\
(9.31)\end{array}$ & $\begin{array}{l}80.00 \\
(8.94)\end{array}$ & $\begin{array}{l}83.33 \\
(9.13)\end{array}$ & $\begin{array}{l}83.33 \\
(9.13)\end{array}$ & 79.26 \\
\hline 5. & Penicillium pinophilum & $\begin{array}{l}76.67 \\
(8.74)\end{array}$ & $\begin{array}{l}83.33 \\
(9.13)\end{array}$ & $\begin{array}{l}80.00 \\
(8.94)\end{array}$ & $\begin{array}{l}80.00 \\
(8.94)\end{array}$ & $\begin{array}{l}96.65 \\
(9.83)\end{array}$ & $\begin{array}{l}86.67 \\
(9.31)\end{array}$ & $\begin{array}{l}83.33 \\
(9.13)\end{array}$ & $\begin{array}{l}83.33 \\
(9.13)\end{array}$ & $\begin{array}{l}86.67 \\
(9.31)\end{array}$ & 84.07 \\
\hline$\overline{6 .}$ & Aspergillus niger & $\begin{array}{l}86.67 \\
(9.31)\end{array}$ & $\begin{array}{l}89.99 \\
(9.48)\end{array}$ & $\begin{array}{l}89.99 \\
(9.48)\end{array}$ & $\begin{array}{l}93.33 \\
(9.66)\end{array}$ & $\begin{array}{l}86.67 \\
(9.31)\end{array}$ & $\begin{array}{l}86.67 \\
(9.31)\end{array}$ & $\begin{array}{l}86.67 \\
(9.31)\end{array}$ & $\begin{array}{l}86.67 \\
(9.31)\end{array}$ & $\begin{array}{l}90.00 \\
(9.49)\end{array}$ & 88.52 \\
\hline 7. & Aspergillus flavus & $\begin{array}{l}80.00 \\
(8.94)\end{array}$ & $\begin{array}{l}83.33 \\
(9.13)\end{array}$ & $\begin{array}{l}76.67 \\
(8.75)\end{array}$ & $\begin{array}{l}86.67 \\
(9.31)\end{array}$ & $\begin{array}{c}76.67 \\
(8.75)\end{array}$ & $\begin{array}{l}86.67 \\
(9.31)\end{array}$ & $\begin{array}{l}83.33 \\
(9.13)\end{array}$ & $\begin{array}{l}80.00 \\
(8.93)\end{array}$ & $\begin{array}{l}83.33 \\
(9.13)\end{array}$ & 81.85 \\
\hline 8. & Drechslera specifera & $\begin{array}{l}83.33 \\
(9.13)\end{array}$ & $\begin{array}{c}8.67 \\
(9.31)\end{array}$ & $\begin{array}{l}83.33 \\
(9.13)\end{array}$ & $\begin{array}{l}80.00 \\
(8.93)\end{array}$ & $\begin{array}{l}83.33 \\
(9.13)\end{array}$ & $\begin{array}{l}86.67 \\
(9.31)\end{array}$ & $\begin{array}{l}83.33 \\
(9.13)\end{array}$ & $\begin{array}{l}83.33 \\
(9.13)\end{array}$ & $\begin{array}{l}83.33 \\
(9.13)\end{array}$ & 83.70 \\
\hline 9. & Penicillium oxalicum & $\begin{array}{l}83.33 \\
(9.13)\end{array}$ & $\begin{array}{l}76.67 \\
(8.75)\end{array}$ & $\begin{array}{l}76.67 \\
(8.75)\end{array}$ & $\begin{array}{l}73.33 \\
(8.54)\end{array}$ & $\begin{array}{c}76.67 \\
(8.75)\end{array}$ & $\begin{array}{l}83.33 \\
(9.13)\end{array}$ & $\begin{array}{l}73.33 \\
(8.54)\end{array}$ & $\begin{array}{l}70.00 \\
(8.35)\end{array}$ & $\begin{array}{l}70.00 \\
(8.35)\end{array}$ & 75.93 \\
\hline 10. & Geotrichum candidum & $\begin{array}{l}86.67 \\
(9.31)\end{array}$ & $\begin{array}{l}76.67 \\
(8.75)\end{array}$ & $\begin{array}{l}83.33 \\
(9.13)\end{array}$ & $\begin{array}{l}80.00 \\
(8.94)\end{array}$ & $\begin{array}{l}83.33 \\
(9.13)\end{array}$ & $\begin{array}{l}76.67 \\
(8.75)\end{array}$ & $\begin{array}{l}83.33 \\
(9.13)\end{array}$ & $\begin{array}{l}80.00 \\
(8.93)\end{array}$ & $\begin{array}{l}80.00 \\
(8.93)\end{array}$ & 81.11 \\
\hline 11. & Culvularia lunata & $\begin{array}{l}80.00 \\
(8.94)\end{array}$ & $\begin{array}{l}83.33 \\
(9.13)\end{array}$ & $\begin{array}{l}80.00 \\
(8.94)\end{array}$ & $\begin{array}{l}83.33 \\
(9.13)\end{array}$ & $\begin{array}{l}80.00 \\
(8.93)\end{array}$ & $\begin{array}{l}86.67 \\
(9.31)\end{array}$ & $\begin{array}{l}80.00 \\
(8.94)\end{array}$ & $\begin{array}{l}86.67 \\
(9.31)\end{array}$ & $\begin{array}{l}83.33 \\
(9.13)\end{array}$ & 82.59 \\
\hline 12. & Aspergillus amstelodami & $\begin{array}{l}80.00 \\
(8.94)\end{array}$ & $\begin{array}{l}83.33 \\
(9.13)\end{array}$ & $\begin{array}{l}86.67 \\
(9.31)\end{array}$ & $\begin{array}{l}83.33 \\
(9.13)\end{array}$ & $\begin{array}{l}80.00 \\
(8.93)\end{array}$ & $\begin{array}{l}86.67 \\
(9.31)\end{array}$ & $\begin{array}{l}80.00 \\
(8.94)\end{array}$ & $\begin{array}{l}80.00 \\
(8.94)\end{array}$ & $\begin{array}{l}86.67 \\
(9.31\end{array}$ & 82.96 \\
\hline 13. & Talaromyces sp. & $\begin{array}{l}83.33 \\
(9.13)\end{array}$ & $\begin{array}{l}80.00 \\
(8.94)\end{array}$ & $\begin{array}{l}86.67 \\
(9.31)\end{array}$ & $\begin{array}{l}83.33 \\
(9.13)\end{array}$ & $\begin{array}{c}86.67 \\
(99.31)\end{array}$ & $\begin{array}{l}86.67 \\
(9.31)\end{array}$ & $\begin{array}{l}83.33 \\
(9.13)\end{array}$ & $\begin{array}{l}80.00 \\
(8.94)\end{array}$ & $\begin{array}{l}83.33 \\
(9.13)\end{array}$ & 83.70 \\
\hline 14. & Chaetomium ochraceum & $\begin{array}{l}93.33 \\
(9.66)\end{array}$ & $\begin{array}{l}86.67 \\
(9.31)\end{array}$ & $\begin{array}{l}86.67 \\
(9.31)\end{array}$ & $\begin{array}{l}80.00 \\
(8.94)\end{array}$ & $\begin{array}{l}86.67 \\
(9.31)\end{array}$ & $\begin{array}{l}86.67 \\
(9.31)\end{array}$ & $\begin{array}{l}83.33 \\
(9.13)\end{array}$ & $\begin{array}{l}83.33 \\
(9.13)\end{array}$ & $\begin{array}{l}86.67 \\
(9.31)\end{array}$ & 85.93 \\
\hline 15. & Control & $\begin{array}{l}86.67 \\
(9.31)\end{array}$ & $\begin{array}{l}86.67 \\
(9.31)\end{array}$ & $\begin{array}{l}86.67 \\
(9.31)\end{array}$ & $\begin{array}{l}83.33 \\
(9.13)\end{array}$ & $\begin{array}{l}83.33 \\
(9.13)\end{array}$ & $\begin{array}{l}83.33 \\
(9.13)\end{array}$ & $\begin{array}{l}86.67 \\
(9.31)\end{array}$ & $\begin{array}{l}86.67 \\
(9.31)\end{array}$ & $\begin{array}{l}86.67 \\
(9.31)\end{array}$ & 85.56 \\
\hline \multirow{2}{*}{\multicolumn{2}{|c|}{ Mean }} & 83.33 & 83.55 & 82.00 & 82.67 & 83.78 & 84.64 & 83.78 & 82.22 & 83.33 & \\
\hline & & \multicolumn{2}{|c|}{ SEm \pm} & $\mathrm{CD}(\mathrm{p}=0.05)$ & \multicolumn{2}{|c|}{ SEm \pm} & $\mathrm{p}=0.05)$ & \multicolumn{2}{|c|}{$\mathrm{SEm} \pm$} & $\mathrm{CD}(\mathrm{p}=0.05)$ & \\
\hline & Concentration (C) & \multicolumn{2}{|c|}{0.05} & NS & \multicolumn{2}{|c|}{0.05} & NS & \multicolumn{2}{|c|}{0.05} & NS & \\
\hline & Treatment (T) & \multicolumn{2}{|c|}{0.12} & .32 & \multicolumn{2}{|c|}{0.12} & 0.33 & \multicolumn{2}{|c|}{0.11} & 0.32 & \\
\hline & $\mathrm{CxT}$ & \multicolumn{2}{|c|}{0.20} & NS & \multicolumn{2}{|c|}{0.20} & 0.57 & \multicolumn{2}{|c|}{0.20} & NS & \\
\hline
\end{tabular}


Table.2 Effect of culture filtrate of fungal endophytes of rice on growth of seedlings (shoots) in vitro

\begin{tabular}{|c|c|c|c|c|c|c|c|c|c|c|c|}
\hline \multirow{3}{*}{\multicolumn{2}{|c|}{ Endophyte species }} & \multicolumn{10}{|c|}{ Growth of roots $(\mathrm{mm})$ of rice seeds treated with culture filtrate of fungal endophytes } \\
\hline & & \multicolumn{3}{|c|}{15 mins } & \multicolumn{3}{|c|}{30 mins } & \multicolumn{3}{|c|}{60 mins } & \multirow[t]{2}{*}{ Mean } \\
\hline & & $25 \%$ & $50 \%$ & $100 \%$ & $25 \%$ & $50 \%$ & $100 \%$ & $25 \%$ & $50 \%$ & $100 \%$ & \\
\hline 1. & Cladosporium cladosporioides & $\begin{array}{c}35.36 \\
(36.48)^{*}\end{array}$ & $\begin{array}{c}39.29 \\
(38.80)\end{array}$ & $\begin{array}{c}36.41 \\
(37.10)\end{array}$ & $\begin{array}{c}36.41 \\
(37.10)\end{array}$ & $\begin{array}{c}36.32 \\
(37.06)\end{array}$ & $\begin{array}{c}34.81 \\
(36.15)\end{array}$ & $\begin{array}{c}41.48 \\
(40.08)\end{array}$ & $\begin{array}{c}36.76 \\
(37.31)\end{array}$ & $\begin{array}{c}33.05 \\
(35.06)\end{array}$ & 36.66 \\
\hline 2. & Penicillium citrinum & $\begin{array}{c}26.23 \\
(30.75)\end{array}$ & $\begin{array}{c}27.97 \\
(31.92)\end{array}$ & $\begin{array}{c}34.15 \\
(35.75)\end{array}$ & $\begin{array}{c}38.85 \\
(38.56)\end{array}$ & $\begin{array}{c}30.70 \\
(33.60)\end{array}$ & $\begin{array}{c}23.96 \\
(29.25)\end{array}$ & $\begin{array}{c}24.67 \\
(29.70)\end{array}$ & $\begin{array}{c}29.69 \\
(32.99)\end{array}$ & $\begin{array}{c}28.75 \\
(32.40)\end{array}$ & 29.44 \\
\hline 3. & Fusarium moniliforme & $\begin{array}{c}32.16 \\
(34.51)\end{array}$ & $\begin{array}{c}40.16 \\
(39.32)\end{array}$ & $\begin{array}{c}32.88 \\
(34.98)\end{array}$ & $\begin{array}{c}42.08 \\
(40.44)\end{array}$ & $\begin{array}{c}38.82 \\
(38.54)\end{array}$ & $\begin{array}{c}39.88 \\
(39.15)\end{array}$ & $\begin{array}{c}28.31 \\
(32.13)\end{array}$ & $\begin{array}{c}32.07 \\
(34.49)\end{array}$ & $\begin{array}{c}15.86 \\
(23.44)\end{array}$ & 33.58 \\
\hline 4. & Trichoderma asperellum & $\begin{array}{c}32.85 \\
(34.93)\end{array}$ & $\begin{array}{c}22.35 \\
(28.19)\end{array}$ & $\begin{array}{c}30.85 \\
(33.72)\end{array}$ & $\begin{array}{c}29.87 \\
(33.12)\end{array}$ & $\begin{array}{c}27.59 \\
(31.55)\end{array}$ & $\begin{array}{c}34.51 \\
(35.97)\end{array}$ & $\begin{array}{c}30.72 \\
(33.56)\end{array}$ & $\begin{array}{c}34.07 \\
(35.71)\end{array}$ & $\begin{array}{c}34.69 \\
(36.08)\end{array}$ & 30.83 \\
\hline 5. & Penicillium pinophilum & $\begin{array}{c}36.92 \\
(37.42)\end{array}$ & $\begin{array}{c}39.80 \\
(39.11)\end{array}$ & $\begin{array}{c}39.83 \\
(39.13)\end{array}$ & $\begin{array}{c}38.08 \\
(38.11)\end{array}$ & $\begin{array}{c}34.22 \\
(35.80)\end{array}$ & $\begin{array}{c}38.86 \\
(36.78)\end{array}$ & $\begin{array}{c}36.27 \\
(37.02)\end{array}$ & $\begin{array}{c}36.41 \\
(37.11)\end{array}$ & $\begin{array}{c}41.71 \\
(40.23)\end{array}$ & 37.68 \\
\hline 6. & Aspergillus niger & $\begin{array}{c}38.60 \\
(38.41)\end{array}$ & $\begin{array}{c}35.08 \\
(36.32)\end{array}$ & $\begin{array}{c}27.15 \\
(30.47)\end{array}$ & $\begin{array}{c}38.61 \\
(38.41)\end{array}$ & $\begin{array}{c}37.40 \\
(37.70)\end{array}$ & $\begin{array}{c}40.87 \\
(39.74)\end{array}$ & $\begin{array}{c}40.01 \\
(39.23)\end{array}$ & $\begin{array}{c}38.09 \\
(38.11)\end{array}$ & $\begin{array}{c}39.34 \\
(38.84)\end{array}$ & 37.24 \\
\hline 7. & Aspergillus flavus & $\begin{array}{c}37.54 \\
(37.79)\end{array}$ & $\begin{array}{c}38.56 \\
(38.39)\end{array}$ & $\begin{array}{c}39.26 \\
(38.80)\end{array}$ & $\begin{array}{c}37.45 \\
(37.73)\end{array}$ & $\begin{array}{c}40.08 \\
(39.27)\end{array}$ & $\begin{array}{c}39.45 \\
(38.90)\end{array}$ & $\begin{array}{c}34.77 \\
(36.13)\end{array}$ & $\begin{array}{c}35.45 \\
(36.54)\end{array}$ & $\begin{array}{c}37.23 \\
(37.30)\end{array}$ & 37.75 \\
\hline 8. & Drechslera specifera & $\begin{array}{c}36.11 \\
(36.92)\end{array}$ & $\begin{array}{c}39.37 \\
(38.86)\end{array}$ & $\begin{array}{c}38.30 \\
(38.23)\end{array}$ & $\begin{array}{c}36.58 \\
(37.21)\end{array}$ & $\begin{array}{c}41.22 \\
(39.93)\end{array}$ & $\begin{array}{c}42.57 \\
(40.73)\end{array}$ & $\begin{array}{c}39.64 \\
(39.01)\end{array}$ & $\begin{array}{c}36.14 \\
(36.95)\end{array}$ & $\begin{array}{c}40.21 \\
(39.35)\end{array}$ & 38.91 \\
\hline 9. & Penicillium oxalicum & $\begin{array}{c}27.39 \\
(31.52)\end{array}$ & $\begin{array}{c}27.39 \\
(31.52)\end{array}$ & $\begin{array}{c}29.20 \\
(32.70)\end{array}$ & $\begin{array}{c}28.07 \\
(31.93)\end{array}$ & $\begin{array}{c}24.63 \\
(29.71)\end{array}$ & $\begin{array}{c}24.72 \\
(29.77)\end{array}$ & $\begin{array}{c}28.85 \\
(32.49)\end{array}$ & $\begin{array}{c}30.13 \\
(33.19)\end{array}$ & $\begin{array}{c}27.77 \\
(31.79)\end{array}$ & 27.57 \\
\hline 10. & Geotrichum candidum & $\begin{array}{c}36.35 \\
(37.06)\end{array}$ & $\begin{array}{c}39.39 \\
(38.87)\end{array}$ & $\begin{array}{c}42.21 \\
(40.51)\end{array}$ & $\begin{array}{c}35.62 \\
(36.63)\end{array}$ & $\begin{array}{c}36.62 \\
(36.63)\end{array}$ & $\begin{array}{c}28.28 \\
(32.12)\end{array}$ & $\begin{array}{c}38.62 \\
(38.42)\end{array}$ & $\begin{array}{c}38.98 \\
(38.63)\end{array}$ & $\begin{array}{c}39.01 \\
(38.65)\end{array}$ & 37.12 \\
\hline 11. & Culvularia lunata & $\begin{array}{c}38.94 \\
(38.61)\end{array}$ & $\begin{array}{c}35.87 \\
(36.79)\end{array}$ & $\begin{array}{c}30.59 \\
(33.55)\end{array}$ & $\begin{array}{c}35.74 \\
(36.71)\end{array}$ & $\begin{array}{c}34.57 \\
(36.00)\end{array}$ & $\begin{array}{c}35.76 \\
(36.72)\end{array}$ & $\begin{array}{c}39.66 \\
(39.03)\end{array}$ & $\begin{array}{c}37.36 \\
(37.67)\end{array}$ & $\begin{array}{c}35.80 \\
(36.74)\end{array}$ & 36.03 \\
\hline 12. & Aspergillus amstelodami & $\begin{array}{c}41.65 \\
(40.19)\end{array}$ & $\begin{array}{c}40.06 \\
(39.26)\end{array}$ & $\begin{array}{c}40.88 \\
(39.74)\end{array}$ & $\begin{array}{c}40.41 \\
(39.46)\end{array}$ & $\begin{array}{c}35.82 \\
(36.74)\end{array}$ & $\begin{array}{c}39.57 \\
(38.96)\end{array}$ & $\begin{array}{c}29.96 \\
(33.17)\end{array}$ & $\begin{array}{c}32.96 \\
(35.03)\end{array}$ & $\begin{array}{c}42.59 \\
(40.73)\end{array}$ & 38.21 \\
\hline 13. & Talaromyces sp. & $\begin{array}{c}33.83 \\
(35.54)\end{array}$ & $\begin{array}{c}31.83 \\
(34.32)\end{array}$ & $\begin{array}{c}37.99 \\
(38.03)\end{array}$ & $\begin{array}{c}37.12 \\
(37.53)\end{array}$ & $\begin{array}{c}35.08 \\
(36.31)\end{array}$ & $\begin{array}{c}39.31 \\
(38.82)\end{array}$ & $\begin{array}{c}38.30 \\
(38.23)\end{array}$ & $\begin{array}{c}35.98 \\
(36.85)\end{array}$ & $\begin{array}{c}36.77 \\
(37.33)\end{array}$ & 36.25 \\
\hline 14. & Chaetomium ochraceum & $\begin{array}{c}32.80 \\
(34.93)\end{array}$ & $\begin{array}{c}34.97 \\
(36.24)\end{array}$ & $\begin{array}{c}36.87 \\
(37.38)\end{array}$ & $\begin{array}{c}36.60 \\
(37.21)\end{array}$ & $\begin{array}{c}37.28 \\
(37.63)\end{array}$ & $\begin{array}{c}35.80 \\
(36.75)\end{array}$ & $\begin{array}{c}36.41 \\
(37.11)\end{array}$ & $\begin{array}{c}38.82 \\
(38.54)\end{array}$ & $\begin{array}{c}38.83 \\
(38.54)\end{array}$ & 36.49 \\
\hline 15. & Control & $\begin{array}{c}42.22 \\
(40.52)\end{array}$ & $\begin{array}{c}42.22 \\
(40.52)\end{array}$ & $\begin{array}{c}42.22 \\
(40.52)\end{array}$ & $\begin{array}{c}38.95 \\
(38.58)\end{array}$ & $\begin{array}{c}38.95 \\
(38.58)\end{array}$ & $\begin{array}{c}38.95 \\
(38.58)\end{array}$ & $\begin{array}{c}41.19 \\
(39.87)\end{array}$ & $\begin{array}{c}41.19 \\
(39.87)\end{array}$ & $\begin{array}{c}41.19 \\
(39.87)\end{array}$ & 40.79 \\
\hline & Mean & 35.26 & 35.62 & 35.92 & 36.70 & 35.22 & 35.13 & 35.62 & 35.61 & 35.52 & \\
\hline & & SEm & & $(\mathrm{p}=0.05)$ & SEn & & $(\mathrm{p}=0.05)$ & SE1 & & $(p=0.05)$ & \\
\hline & Concentration (C) & 0.03 & & NS & 0.0 & & NS & 0.1 & & NS & \\
\hline & Treatment (T) & 0.08 & & 0.21 & 0.0 & & 0.24 & 0.0 & & 0.21 & \\
\hline & Cx T & 0.13 & & 0.37 & 0.1 & & 0.42 & 0.1 & & 0.36 & \\
\hline
\end{tabular}


Table.3 Effect of culture filtrate of fungal endophytes of rice on growth of seedlings (roots) in vitro

\begin{tabular}{|c|c|c|c|c|c|c|c|c|c|c|c|}
\hline \multirow{3}{*}{\multicolumn{2}{|c|}{ Endophyte species }} & \multicolumn{10}{|c|}{ Growth of roots $(\mathrm{mm})$ of rice seeds treated with culture filtrate of fungal endophytes } \\
\hline & & \multicolumn{3}{|c|}{15 mins } & \multicolumn{3}{|c|}{30 mins } & \multicolumn{3}{|c|}{$60 \mathrm{mins}$} & \multirow[t]{2}{*}{ Mean } \\
\hline & & $25 \%$ & $50 \%$ & $100 \%$ & $25 \%$ & $50 \%$ & $100 \%$ & $25 \%$ & $50 \%$ & $100 \%$ & \\
\hline 1. & Cladosporium cladosporioides & $\begin{array}{c}35.36 \\
(36.48)^{*}\end{array}$ & $\begin{array}{c}39.29 \\
(38.80)\end{array}$ & $\begin{array}{c}36.41 \\
(37.10)\end{array}$ & $\begin{array}{c}36.41 \\
(37.10)\end{array}$ & $\begin{array}{c}36.32 \\
(37.06)\end{array}$ & $\begin{array}{c}34.81 \\
(36.15)\end{array}$ & $\begin{array}{c}41.48 \\
(40.08)\end{array}$ & $\begin{array}{c}36.76 \\
(37.31)\end{array}$ & $\begin{array}{c}33.05 \\
(35.06)\end{array}$ & 36.66 \\
\hline 2. & Penicillium citrinum & $\begin{array}{c}26.23 \\
(30.75)\end{array}$ & $\begin{array}{c}27.97 \\
(31.92)\end{array}$ & $\begin{array}{c}34.15 \\
(35.75)\end{array}$ & $\begin{array}{c}38.85 \\
(38.56)\end{array}$ & $\begin{array}{c}30.70 \\
(33.60)\end{array}$ & $\begin{array}{c}23.96 \\
(29.25)\end{array}$ & $\begin{array}{c}24.67 \\
(29.70)\end{array}$ & $\begin{array}{c}29.69 \\
(32.99)\end{array}$ & $\begin{array}{c}28.75 \\
(32.40)\end{array}$ & 29.44 \\
\hline 3. & Fusarium moniliforme & $\begin{array}{c}32.16 \\
(34.51)\end{array}$ & $\begin{array}{c}40.16 \\
(39.32)\end{array}$ & $\begin{array}{c}32.88 \\
(34.98)\end{array}$ & $\begin{array}{c}42.08 \\
(40.44)\end{array}$ & $\begin{array}{c}38.82 \\
(38.54)\end{array}$ & $\begin{array}{c}39.88 \\
(39.15)\end{array}$ & $\begin{array}{c}28.31 \\
(32.13)\end{array}$ & $\begin{array}{c}32.07 \\
(34.49)\end{array}$ & $\begin{array}{l}15.86 \\
(23.44)\end{array}$ & 33.58 \\
\hline$\overline{4 .}$ & Trichoderma asperellum & $\begin{array}{c}32.85 \\
(34.93)\end{array}$ & $\begin{array}{c}22.35 \\
(28.19)\end{array}$ & $\begin{array}{c}30.85 \\
(33.72)\end{array}$ & $\begin{array}{c}29.87 \\
(33.12)\end{array}$ & $\begin{array}{c}27.59 \\
(31.55)\end{array}$ & $\begin{array}{c}34.51 \\
(35.97)\end{array}$ & $\begin{array}{c}30.72 \\
(33.56)\end{array}$ & $\begin{array}{c}34.07 \\
(35.71)\end{array}$ & $\begin{array}{c}34.69 \\
(36.08)\end{array}$ & 30.83 \\
\hline 5. & Penicillium pinophilum & $\begin{array}{c}36.92 \\
(37.42)\end{array}$ & $\begin{array}{c}39.80 \\
(39.11)\end{array}$ & $\begin{array}{c}39.83 \\
(39.13)\end{array}$ & $\begin{array}{c}38.08 \\
(38.11)\end{array}$ & $\begin{array}{c}34.22 \\
(35.80)\end{array}$ & $\begin{array}{c}38.86 \\
(36.78)\end{array}$ & $\begin{array}{c}36.27 \\
(37.02)\end{array}$ & $\begin{array}{c}36.41 \\
(37.11)\end{array}$ & $\begin{array}{c}41.71 \\
(40.23)\end{array}$ & 37.68 \\
\hline 6. & Aspergillus niger & $\begin{array}{c}38.60 \\
(38.41)\end{array}$ & $\begin{array}{c}35.08 \\
(36.32)\end{array}$ & $\begin{array}{c}27.15 \\
(30.47)\end{array}$ & $\begin{array}{c}38.61 \\
(38.41)\end{array}$ & $\begin{array}{c}37.40 \\
(37.70)\end{array}$ & $\begin{array}{c}40.87 \\
(39.74)\end{array}$ & $\begin{array}{c}40.01 \\
(39.23)\end{array}$ & $\begin{array}{c}38.09 \\
(38.11)\end{array}$ & $\begin{array}{c}39.34 \\
(38.84)\end{array}$ & 37.24 \\
\hline 7. & Aspergillus flavus & $\begin{array}{c}37.54 \\
(37.79)\end{array}$ & $\begin{array}{c}38.56 \\
(38.39)\end{array}$ & $\begin{array}{c}39.26 \\
(38.80)\end{array}$ & $\begin{array}{c}37.45 \\
(37.73)\end{array}$ & $\begin{array}{c}40.08 \\
(39.27)\end{array}$ & $\begin{array}{c}39.45 \\
(38.90)\end{array}$ & $\begin{array}{c}34.77 \\
(36.13)\end{array}$ & $\begin{array}{c}35.45 \\
(36.54)\end{array}$ & $\begin{array}{c}37.23 \\
(37.30)\end{array}$ & 37.75 \\
\hline 8. & Drechslera specifera & $\begin{array}{c}36.11 \\
(36.92)\end{array}$ & $\begin{array}{c}39.37 \\
(38.86)\end{array}$ & $\begin{array}{c}38.30 \\
(38.23)\end{array}$ & $\begin{array}{c}36.58 \\
(37.21)\end{array}$ & $\begin{array}{c}41.22 \\
(39.93)\end{array}$ & $\begin{array}{c}42.57 \\
(40.73)\end{array}$ & $\begin{array}{c}39.64 \\
(39.01)\end{array}$ & $\begin{array}{c}36.14 \\
(36.95)\end{array}$ & $\begin{array}{c}40.21 \\
(39.35)\end{array}$ & 38.91 \\
\hline 9. & Penicillium oxalicum & $\begin{array}{c}27.39 \\
(31.52)\end{array}$ & $\begin{array}{c}27.39 \\
(31.52)\end{array}$ & $\begin{array}{c}29.20 \\
(32.70)\end{array}$ & $\begin{array}{c}28.07 \\
(31.93)\end{array}$ & $\begin{array}{c}24.63 \\
(29.71)\end{array}$ & $\begin{array}{c}24.72 \\
(29.77)\end{array}$ & $\begin{array}{c}28.85 \\
(32.49)\end{array}$ & $\begin{array}{c}30.13 \\
(33.19)\end{array}$ & $\begin{array}{c}27.77 \\
(31.79)\end{array}$ & 27.57 \\
\hline 10. & Geotrichum candidum & $\begin{array}{c}36.35 \\
(37.06)\end{array}$ & $\begin{array}{c}39.39 \\
(38.87)\end{array}$ & $\begin{array}{c}42.21 \\
(40.51)\end{array}$ & $\begin{array}{c}35.62 \\
(36.63)\end{array}$ & $\begin{array}{c}36.62 \\
(36.63)\end{array}$ & $\begin{array}{c}28.28 \\
(32.12)\end{array}$ & $\begin{array}{c}38.62 \\
(38.42)\end{array}$ & $\begin{array}{c}38.98 \\
(38.63)\end{array}$ & $\begin{array}{c}39.01 \\
(38.65)\end{array}$ & 37.12 \\
\hline 11. & Culvularia lunata & $\begin{array}{c}38.94 \\
(38.61)\end{array}$ & $\begin{array}{c}35.87 \\
(36.79)\end{array}$ & $\begin{array}{c}30.59 \\
(33.55)\end{array}$ & $\begin{array}{c}35.74 \\
(36.71)\end{array}$ & $\begin{array}{c}34.57 \\
(36.00)\end{array}$ & $\begin{array}{c}35.76 \\
(36.72)\end{array}$ & $\begin{array}{c}39.66 \\
(39.03)\end{array}$ & $\begin{array}{c}37.36 \\
(37.67)\end{array}$ & $\begin{array}{c}35.80 \\
(36.74)\end{array}$ & 36.03 \\
\hline 12. & Aspergillus amstelodami & $\begin{array}{c}41.65 \\
(40.19)\end{array}$ & $\begin{array}{c}40.06 \\
(39.26)\end{array}$ & $\begin{array}{c}40.88 \\
(39.74)\end{array}$ & $\begin{array}{c}40.41 \\
(39.46)\end{array}$ & $\begin{array}{c}35.82 \\
(36.74)\end{array}$ & $\begin{array}{c}39.57 \\
(38.96)\end{array}$ & $\begin{array}{c}29.96 \\
(33.17)\end{array}$ & $\begin{array}{c}32.96 \\
(35.03)\end{array}$ & $\begin{array}{c}42.59 \\
(40.73)\end{array}$ & 38.21 \\
\hline 13. & Talaromyces sp. & $\begin{array}{c}33.83 \\
(35.54)\end{array}$ & $\begin{array}{c}31.83 \\
(34.32)\end{array}$ & $\begin{array}{c}37.99 \\
(38.03)\end{array}$ & $\begin{array}{c}37.12 \\
(37.53)\end{array}$ & $\begin{array}{c}35.08 \\
(36.31)\end{array}$ & $\begin{array}{c}39.31 \\
(38.82)\end{array}$ & $\begin{array}{c}38.30 \\
(38.23)\end{array}$ & $\begin{array}{c}35.98 \\
(36.85)\end{array}$ & $\begin{array}{c}36.77 \\
(37.33)\end{array}$ & 36.25 \\
\hline 14. & Chaetomium ochraceum & $\begin{array}{c}32.80 \\
(34.93)\end{array}$ & $\begin{array}{c}34.97 \\
(36.24)\end{array}$ & $\begin{array}{c}36.87 \\
(37.38)\end{array}$ & $\begin{array}{c}36.60 \\
(37.21)\end{array}$ & $\begin{array}{c}37.28 \\
(37.63)\end{array}$ & $\begin{array}{c}35.80 \\
(36.75)\end{array}$ & $\begin{array}{c}36.41 \\
(37.11)\end{array}$ & $\begin{array}{c}38.82 \\
(38.54)\end{array}$ & $\begin{array}{c}38.83 \\
(38.54)\end{array}$ & 36.49 \\
\hline & Control & $\begin{array}{c}42.22 \\
(40.52)\end{array}$ & $\begin{array}{c}42.22 \\
(40.52)\end{array}$ & $\begin{array}{c}42.22 \\
(40.52)\end{array}$ & $\begin{array}{c}38.95 \\
(38.58)\end{array}$ & $\begin{array}{c}38.95 \\
(38.58)\end{array}$ & $\begin{array}{c}38.95 \\
(38.58)\end{array}$ & $\begin{array}{c}41.19 \\
(39.87)\end{array}$ & $\begin{array}{c}41.19 \\
(39.87)\end{array}$ & $\begin{array}{c}41.19 \\
(39.87)\end{array}$ & 40.79 \\
\hline & Mean & 35.26 & 35.62 & 35.92 & 36.70 & 35.22 & 35.13 & 35.62 & 35.61 & 35.52 & \\
\hline & & SEm & & $p=0.05)$ & SEn & & $(p=0.05)$ & $S E l$ & & $(p=0.05)$ & \\
\hline & Concentration (C) & 0.3 & & NS & 0.3 & & NS & 0.3 & & NS & \\
\hline & Treatment (T) & 0.8 & & 2.27 & 0.6 & & 1.94 & 0.7 & & 1.99 & \\
\hline & $\mathrm{Cx} \mathrm{T}$ & 1.4 & & 3.94 & 1.1 & & 3.35 & 1.2 & & 3.45 & \\
\hline
\end{tabular}


The Penicillium spp. as endophytes may also produce metabolites in their culture filtrates that would give protection to the rice seeds from harmful seed microflora at the time of germination (Saikkonen et al., 2004b).

\section{Seedling growth}

Growth of seedlings (shoots) of rice seeds treated with culture filtrate of fungal endophytes

It is evident from the datas from Table 2 that various treatments with culture filtrates at different period of dipping showed significant influence on the growth of shoots. The maximum shoot length was recorded with seeds treated with $C$. cladosporioides $(13.74$ $\mathrm{mm}$ ) when dipped for 30 minutes at $100 \%$ concentration as compared to $6.74 \mathrm{~mm}$ shoot length recorded in control experiments. The least shoot length of rice seed was recorded with endophyte $G$. candidum $(3.02 \mathrm{~mm})$ at $25 \%$ concentration dipped for 15 minutes. The highest mean shoot length was recorded with rice seeds dipped in culture filtrate of $C$. cladosporioides (11.29) and the least mean shoot length was recorded in the rice seeds dipped in that of G. candidum (3.68) as compared to control that recorded $6.76 \mathrm{~mm}$ shoot length. Whereas, with respect to time and concentration, irrespective of the different culture filtrates of endophytes applied, the rice seeds dipped in 50\% concentration of culture filtrate for 30 minutes recorded the highest mean shoot length $(8.04 \mathrm{~mm})$ and the least mean shoot length (7.33) was recorded for rice seeds dipped in $100 \%$ concentration culture filtrates dipped for 30 minutes. Statistical analysis revealed that different concentrations of the fungal endophytes viz. 25,50 and $100 \%$ had no significant influence of on the length of shoot of rice seeds at different time intervals of 15,30 and 60 minutes. On the contrary, treatments with different fungal endophyte species and interactions between treatments applied and concentrations of the culture filtrate were found to have significant effect on the growth of shoots of rice seeds.

Growth of seedlings (roots) of rice seeds treated with culture filtrate of fungal endophytes

The datas pertaining to the effect of culture filtrate of fungal endophytes on the growth of roots of rice seeds are presented in Table 3 . The maximum root length was recorded with seeds treated with A. amstelodami (42.59 $\mathrm{mm}$ ) when dipped for 60 minutes at $100 \%$ concentration of culture filtrate as compared to $41.19 \mathrm{~mm}$ of root length recorded in control. The minimum root length was recorded with seeds dipped in $F$. moniliforme (15.86) at $100 \%$ concentration dipped for 60 minutes. The highest mean root length was recorded with rice seeds dipped in culture filtrate of D. specifera (38.91) and the least mean root length was recorded in the rice seeds dipped in that of $T$. asperellum (30.83) as compared to control that recorded 40.79 $\mathrm{mm}$ root length. Whereas, with respect to time and concentration, irrespective of the different culture filtrates of endophytes applied, the rice seeds dipped in $100 \%$ concentration of culture filtrate for 15 minutes recorded the highest mean root length (35.92) and the least mean root length (35.13) was recorded for rice seeds dipped in $100 \%$ concentration culture filtrates dipped for 30 minutes. No significant influence was observed for different concentrations of the fungal endophytes at different time intervals. On the contrary, treatments with different fungal endophyte species and interactions between treatments applied and concentrations of the culture filtrate were found to have significant effect on the growth of shoots of rice seeds.

Earlier works suggests that endophyte species have positive effect on the growth of 
seedlings (Bandar et al., 2006, Lin et al., 2007, Bo et al., 2014 and $\mathrm{Na}$ et al., 2014). The present findings are also in conformity with similar explanation. Endophytic species like Phomopsis sp. (Lin et al., 2007, Chao et al., 2008 and Bo et al., 2014) have already been shown to promote the growth of rice plant. Kumar et al., (2012) reported that culture filtrate of Piriformospora indica (a root endophytic fungus) had significant enhancement in lignan production and growth of Linum album hairy root cultures. Bagheri et al., (2013) also reported that endophytic fungus, Piriformospora indica has a pronounced growth-promoting activity on rice plant. The growth of seedlings upon treatment with different culture filtrate of fungal endophytes may be due to the increment in the production lignans like podophyllotoxin and 6 -methoxypodophyllotoxin concentratation which also coincides with the increase in phenylalanine ammonia lyase activity in the rice plant (Kumar et al., 2012). Bagheri et al., (2013) reported that endophytes increase the biomass of aerial parts and root, total soluble proteins, Relative Water Content (RWC), free proline content and enzyme antioxidants activity of inoculated rice as compared to un-inoculated ones. Since the data obtained in the present investigation also indicates significant increase in the growth of seedlings (shoot), thus it corroborates with the findings of the earlier workers.

With respect to root length of rice seedlings, the mean root length was shorter in all the treatments with culture filtrate of fungal endophytes than that of control. This indicates that seed treatment with culture filtrate of endophytes may not be a viable idea to obtain increased root length that would actually reflect increased plant establishment in an area. As our experiment was limited to only five days after germination and endophytic fungi were not directly involved, and there was a mixtures of fungal metabolites in their culture filtrate, effect of endophytes on rice root length was perhaps not translated well. Other workers have reported growth promoting effect of various fungal endophytes viz. P. indica on rice plant (Bagheri et al., 2013) and Linum album (Kumar et al., 2012). $P$. indica is a root endophytic fungus. Endophytic fungi Phoma glomerata and Penicillium sp. significantly promoted shoot growth in gibberellic acid deficient dwarf mutant variety of rice indicating role of endophytes in producing plant growth hormone like GA that is known for inducing shoot growth (Waqas et al.,2012).

The present study manifests the effects of fungal endophytes on the germination of rice seedlings as well as shoot and root length of rice seedlings upto five days after germination. In order to observe the full beneficial effects of fungal endophytes, a further elaborative study is required to be taken up till maturity stage of rice plant including the biochemical analysis of culture filtrate of the endophytes. Individual endophytes may be screened for their beneficial effects on rice plant and further explored for commercialization.

\section{References}

Anonymous. 2009. Brookhaven National Laboratory and their Belgian colleagues at Hasslet University. Applied and Environmental Microbiology. Retrieved 24 July 2012.

Arnold, A, E., Meffa, L. C., Kyollo, D., Rojas, E. I., Maynard, Z., Robbins, N and Herre, E. A. 2003. Fungal endophytes limit pathogen damage in a tropical tree. Proceedings of the National. Academy of Sciences. USA 100: 15649-15654.

Bagheri, A. A., Saadatmand, S., Niknam, V., Nejadsatari, T and Babaeizad, V. 2013. 
Effect of endophytic fungus, Piriformospora indica, on growth and activity of antioxidant enzymes of rice (Oryza sativa L.) under salinity stress. International journal of Advanced Biological and Biomedical Research 1 (11): 1337-1350

Bandara, W. M. M. S., Seneviratne, Gamini and Kulasooriya, S. A. 2006. Interactions among endophytic bacteria and fungi: Effects and potentials. Journal of Biosciences 31(5):645-650.

Bo, Y., Mi, W. X., Yan, M. H., Yong, J., Xia, L and Chao, D, C. 2014. Effects of the fungal endophyte Phomopsis

liquidambari on nitrogen uptake and metabolism in rice. Plant Growth Regulation 73(2):165-179.

Bush, L. P., Wilkinson, H, H and Schardl, C. L. 1997. Bioprotective alkaloids of grass-fungal endophyte symbioses. Plant Physiology 114:1- 7.

Chao, D. C., Lin, Z, Y., Yin, Y. Q., Shan, S. $\mathrm{Q}$ and Xia, L. (2008). The effects of increasing production of endophytes Phomopsis B3 on rice. Agricultural Science \& Technology - Hunan 9(1):3942.

Chapela, I. H. 1989. Fungi in health stems and American beech and aspen: a comparative study. New Phytologist 113:65- 75 .

Cheplick, G. P., Clay, K and Wray, S. 1989. Interactions between fungal endophyte infection and nutrient limitation in the grasses Lolium perenne and Festuca arundinacea. New Phytologist 111:899727.

Clay, K and Holah, J. 1999. Fungal endophyte symbiosis and plant diversity in successional fields. Science 285: 17421744.

Clay, K and Schardl, C. 2002. Evolutionary origins and ecological consequences of endophyte symbiosis with grasses. American Naturalist 160: 99-127.
Clay, K. 1987. Effects of fungal en-dophytes on the seed and seedling biolo-gy of Lolium perenne and Festuca arundinacea. Oecologia 73:358-62.

Compant, S., Reiter, B., Sessitsch, A., Nowak, J., Clément, C and Barka, E. A. 2005. Endophytic colonization of Vitis vinifera $\mathrm{L}$. by plant growth- promoting bacterium Burkholderia sp. strain PsJN. Applied and Environmental Microbiology 71:1685-1693.

Don, C. 2011. Dairy Farmer Finds Unusual Forage Grass. Casler. USDA. Retrieved August 1, 2012.

Ezra, D., Hess, W. M and Strobel, G. A. 2004. New endophytic isolates of Muscodor albus, a volatile-antibioticproducing fungus. Microbiology 150: 4023-4031.

Faeth, S. H and Fagan, W. F. 2002. Fungal endophytes: Common host plant symbionts but uncommon mutualists. Integrative and Comparative Biology 42(2): 360-368.

Faeth, S. H and Hammon, K. E. 1997. Fungal endophytes in oak trees. Long-term patterns of abundance and associations with leafminers. Ecology 78:810- 819.

Fisher, P. J and Petrini, O. 1992. Fungal saprobes and pathogens as endophytes of rice (Oryza sativa L.). New Phytologist 120(1):137-143.

Gunatilaka, A. A. L. 2006. Natural products from plant-associated microorganisms: Distribution, structural diversity, bioactivity and implication of their occurance. Journal of Natural Products. 69: 509-526.

Höflich, G. 2000. Colonization and growth promotion of nonlegumes by Rhizobium bacteria. Microbial Biosystems: New Frontiers; in Proceedings of the 8th International Symposium on Microbial Ecology (eds) C R Bell, M., Brylinsky and Johnson-Green, P. (Halifax: 
Atlantic Canada Society for Microbial Ecology). pp 827-830.

Imchen, R. 2015. Fungal endophytes of rice. M.Sc Thesis. Department of Plant Pathology, SASRD, Nagaland University.

Johri, B. N. 2006. Endophytes to the rescue of plants. Current Science 90: 1315-1316.

Kumar, V., Rajauria, G., Sahai, V and Bisaria, V. S. 2012. Culture filtrate of root endophytic fungus Piriformospora indica promotes the growth and lignan production of Linum album hairy root cultures. Process Biochemistry 47: 901907

Lin, Y. Z., Chao, D. C., Xia, L., Shuang, T. L and Xiang, W. X. 2007. Extensive host range of an endophytic fungus affects the growth and physiological functions in rice (Oryza sativa L.). Symbiosis (Rehovot) 43(1):21-28.

Na, W., Zhen, Y., Jun, C., Xun, C., Na, G and Tao, Y. 2014. Effects of extracts of plant endophyte on growth of rice. Journal of Jilin Agricultural University 36(1): 10-16.

Saikkonen, K., Helander, M and Faeth, S. H. 2004a. Fungal endophytes: hitchhikers of the green world. Plant microbiology (eds) Gillings, M. and Holmes, A. (Oxford: BIOS Scientific Publishers). pp 77-95.

Saikkonen. K., Wa, L. P., Helander, M and Faeth, S. H. 2004b. Evolution of endophyte-plant symbioses. Trends in Plant Science 9:275-280.

Schulthess, F and Faeth, S. H. 1998. Distribution, abundances and associations of the endophytic fungal community of Arizona fescue (Festuca arizonica). Mycologia 90: 569- 578.

Suryanarayanan, T. S., Wittlinger, S., Faeth, S. H. 2005. Endophytic fungi associated with cacti of Arizona. Mycological Research 109: 635- 639.

Taechowisan, T., Chunhua, L. U., Shen, Y and Lumyong, S. 2005. Secondary metabolites from endophytic Streptomyces aureofaciens CMUAc130 and their antifungal activity. Microbiology 151: 1691-1695.

Tian, X. L., Cao, L. X., Tan, H. M., Zeng, Q. G., Jia, Y. Y., Han, W. Q and Zhou, S. N. 2004. Study on the communities of endophytic fungi and endophytic actinomycetes from rice and their antipathogenic activities in vitro. World Journal of Microbiology \& Biotechnology 20(3):303-309.

Waqas, M., Khan, A. L., Kamran, M., Hamayun, M., Kang, S. M., Kim, Y. H and Lee, I. J. 2012. Endophytic fungi produce Gibberellins and Indoleacetic acid and promotes host-plant growth during stress. Molecules 17: 1075410773.

Wilson, D. 1993. Fungal endophytes: out of sight but should not be out of mind? Oikos 68: 379-384.

\section{How to cite this article:}

Lalngaihawmi, S. Banik, P. Chakruno and Khatemenla. 2018. Effect of Rice Fungal Endophytes on Seed Germination and Seedling Growth of Rice. Int.J.Curr.Microbiol.App.Sci. 7(04): 3653-3663. doi: https://doi.org/10.20546/ijcmas.2018.704.411 